\title{
Surgical site infections causes and the burden of Staphylococcus aureus in these infections with their antibiotic susceptibility pattern
}

\author{
Kavitha Kannaiyan ${ }^{1}$, Latha Ragunathan ${ }^{1 *}$ and Kanniappan Venkatachalam ${ }^{2}$ \\ ${ }^{1}$ Department of Microbiology, Aarupadai Veedu Medical College \& Hospital, Puducherry. India \\ ${ }^{2}$ Department of Surgery, Aarupadai Veedu Medical College \& Hospital, Puducherry. India
}

\begin{abstract}
Background: Surgical site infections (SSI) set hurdles in the healing course of a significant proportion of abdominal surgical patients and thus associated with excessive health care costs. Staphylococcus aureus remains the principal causative agent in these infections. Also the emergence of multidrug resistant bacterial strains further complicates the recovery course of the patients and thus adds burden to both the patients and the surgeon.

The intention of this study is to evaluate the etiology of SSI, to establish the incidence of S aureus and to determine their antimicrobial susceptibility pattern in patients with SSI.

Methods: A cross section study was done in Aarupadai veedu medical college in patients attending surgery outpatient department for SSI. Purulent material was collected either with syringe or 2 sterile swabs and was sent to the microbiology department. The sample was processed as per standard guidelines, identified and antibiotic susceptibility was done for S.aureus.

Result: Among the 63 SSI patients, 50 (79.4\%) were culture positive. The most frequently isolated organism was S aureus 23 (36.5\%) isolates. Among them methicillin resistant S.Aureus (MRSA) was 9 (39.1\%). Both Methicillin sensitive S.aureus (MSSA) and MRSA were all sensitive to linezolid and vancomycin. Majority of the isolates were sensitive to amikacin and clindamicin.

Conclusion: Thus our study concludes S. aureus as the foremost etiological agent of SSI, and treatment is further complicated by emerging multidrug resistant MRSA where empirical therapy can be started with clindamicin, amikacin in moderate risk cases and linezolid, vancomycin in high risk cases.
\end{abstract}

Keywords: Abdominal Surgical Patients, Methicillin Resistant Staphylococcus Aureus, Multidrug Resistance

\section{Introduction}

Postoperative wound infections obscure the recovery course of many patients ${ }^{[1]}$ and thus a nightmare to the surgeons. Also the increase in surgical procedures will contribute to more surgical site infections, and thus worth further awareness ${ }^{[2]}$. As per the statement of Centers for disease control and prevention (CDC) SSI accounts for $22 \%$ of all health care associated infections ${ }^{[3]}$.

Microbial contamination is inevitable during surgery, as skin incision itself disrupts the skin barrier ${ }^{[4]}$. SSI is a consequence of microorganisms inoculated into the wound at the time of surgery. Pathogens that contaminate surgical site can be part of patient's endogenous flora or acquired from the hospital environment (exogenous source) ${ }^{[5]}$. Incision by virtue impairs first line of defense between environmental microbes and internal host environment; thereby the exposed tissues are at risk of contamination with patient's endogenous flora ${ }^{[6]}$. Among the various pathogens Staphylococcus aureus is the most important pathogen in SSI around the world ${ }^{[3]}$. Infections caused by this pathogen ranges from superficial to deep seated. It may even cause severe life threatening complications in orthopaedic and cardiac surgery ${ }^{[2]}$.

Particular concern in the infections caused by S.aureus is due to its increasing resistance to methicillin ${ }^{[7]}$. MRSA now turns out to be the bug bear of modern surgical practice. Its increased prevalence in hospitalized patients and moreover the emergence of vancomycin resistance makes it mandatory to control the spread of MRSA infection [8]. The rationale of the present work was to establish the aetiological causes of surgical site infections, the incidence of Staphylococcal infections among them and also to analyze the sensitivity pattern.

\section{Materials And Methods}

Study Population And Area: This study was carried out in the department of microbiology Aarupadai Veedu medical college and hospital, Puducherry from the year 2015- 
2016 after getting approval from the ethical committee. Patients with post operative wound infections with signs of skin inflammation and abscess or drainage at the wound site were enrolled in the study after consent. The patients included both our patients who had undergone surgery in our college and also patients attending our surgery department for SSI treatment who had undergone surgery elsewhere. Patient who were on antibiotics were excluded from our study.

Microbioloical Investigation: Purulent material of $0.5 \mathrm{ml}$ to $1.0 \mathrm{ml}$ was collected wherever possible with syringe. When discharge was minimal it was collected using two sterile swabs without contaminating them with skin commensals. The samples were sent to microbiology department at the earliest. Gram stain for microscopy was done and then the samples were inoculated in blood agar, Mac Conkey agar and were incubated overnight. Sterile samples with more than 7 days infection were further screened for acid fast bacilli by Ziehl Neelsen stain and the culture plates were further incubated for 7 days. The organisms were identified based on their colony appearance, gram stain and biochemical tests as per standard procedures ${ }^{[9]}$. Antibiotic susceptibility tests were done by Kirby Bauer disc diffusion method following Clinical and Laboratory Standards Institute (CLSI) guidelines ${ }^{[10]}$.

The colonies suggestive of Staphylococcus aureus were identified by standard procedures. Tube coagulase was taken as the main criteria for identification of Staphylococcus aureus. Antibiotic susceptibility testing was done using commercially available Penicillin (10units), Linezolid $(30 \mu \mathrm{g})$, Clindamycin $(2 \mu \mathrm{g}), \quad$ Erythromycin $(15 \mu \mathrm{g})$, Ciprofloxacin $(5 \mu \mathrm{g})$, ceftriaxone $(30 \mu \mathrm{g})$, Co-trimoxazole $(1.25 / 23.75 \mu \mathrm{g})$, mikacin $(30 \mu \mathrm{g})$, and Cefoxitin $(30 \mu \mathrm{g})$. Vancomycin sensitivity was done using screening agar. All Staphylococcus aureus strains were screened for
MRSA by detection of resistance to Cefoxitin disc (zone of inhibition was $\leq 21 \mathrm{~mm}$ ) following the CLSI guidelines. Staphylococcus aureus ATCC 25923 was used as control strain.

\section{Result}

Among the 63 established post operative wound infections (Table 1) treated in our surgery department, 44 (69.8\%) patients were females and $19(30.5 \%)$ patients were males, their mean age was 32.35 with standard deviation 13.35. Fifty patients $(79.4 \%)$ were culture positive and among them 47 (94\%) were acute pyogenic organisms. Rapidly growing Mycobacteria was grown in $3(6 \%)$ patients who had chronic postoperative wound infections of more than 7 days and acid fast bacilli positive. No organisms were isolated in 13 patients $(20.6 \%)$. The most frequently isolated organism was S. aureus $23(36.5 \%)$ isolates. Other organisms isolated were Escherichia coli, Pseudomonas aeruginosa, Enterococcus faecalis, Staphylococcus epidermidis, Klebsiella pneumonia, Acinetobacter species shown in (Table 2).

S. aureus which was isolated in $14(60.9 \%)$ wound samples were sensitive to cephoxitin. All the isolates were also sensitive to linezolid and vancomycin $14(100 \%)$. Their sensitivity for other antibiotics were amikacin 13(92.9\%), clindamycin $12(85.7 \%)$, cotrimoxazole 9 (64.3\%), erythromycin $8(57.1 \%)$, ciprofloxacin $4(28.6 \%)$, ceftriaxone $2(14.3 \%) \%$ and least pencillin 1(7\%) [Table 3].

Methicillin resistant S.aureus was isolated in 9 (39.1\%) wound samples. All the MRSA isolated were however sensitive to linezolid and vancomycin $9(100 \%)$. Their sensitivity for other antibiotics were as follows clindamycin $7(77.8 \%)$, amikacin $8(88.9 \%)$, cotrimoxazole $4(44.4 \%)$, erythromycin $3(33.3 \%)$ and ciprofloxacin2 (22.2\%) [Table 3].

Table 1: Type of surgery.

\begin{tabular}{|c|c|c|}
\hline 1. & Caeserean section & $\mathbf{2 5}$ \\
\hline 2. & Hystrectomy & 13 \\
\hline 3 & Appendicectomy & 11 \\
\hline 4. & Herniotomy & 4 \\
\hline 5. & Cholecystectomy & 1 \\
\hline 6. & Pyeloplasty & $\mathbf{6 3}$ \\
\hline
\end{tabular}

Table 2: Prevalence of aerobic organisms from surgical site infections.

\begin{tabular}{|c|c|c|}
\hline Organisms & No of isolates $\mathbf{n = 5 0}$ & Percentage \\
\hline Staphylococcus aureus + MRSA & 23 & $36.5 \%$ \\
\hline Escherichia coli & 8 & $12.7 \%$ \\
\hline
\end{tabular}




\begin{tabular}{|c|c|c|}
\hline Organisms & No of isolates $\mathbf{n}=\mathbf{5 0}$ & Percentage \\
\hline Pseudomonas aeruginosa & 6 & $9.5 \%$ \\
\hline Enterococcus faecalis & 4 & $4.3 \%$ \\
\hline Staphylococcus epidermidis & 3 & $4.7 \%$ \\
\hline Mycobacterium fortuitum & 3 & $3.2 \%$ \\
\hline Klebsiella pneumoniae & 2 & $1.6 \%$ \\
\hline Acinetobacter species & 1 & $\mathbf{7 9 . 2 \%}$ \\
\hline Total & $\mathbf{5 0}$ & \\
\hline
\end{tabular}

Table 3: Antimicrobial sensitivity patterns of methicillin-resistant S. Aureus (mrsa) and methicillin susceptible S.aureus (mssa) strains (\% sensitivity) isolated from SSI.

\begin{tabular}{|c|c|c|}
\hline Drugs & MRSA susceptible $\%(\mathbf{n = 9})$ & MSSA susceptible \% $(\mathbf{n}=\mathbf{1 4})$ \\
\hline Cephoxitin & 0 & $14(100 \%)$ \\
\hline Penicillin & 0 & $1(7 \%)$ \\
\hline Ceftriaxone & 0 & $2(14.3 \%)$ \\
\hline Ciprofloxacin & $2(22.2 \%)$ & $4(28.6 \%)$ \\
\hline Clindamycin & $7(77.8 \%)$ & $12(85.7 \%)$ \\
\hline Co trimoxazole & $4(44.4 \%)$ & $9(64.3 \%)$ \\
\hline Amikacin & $8(88.9 \%)$ & $13(92.9 \%)$ \\
\hline Erythromicin & $3(33.3 \%)$ & $8(57.1 \%)$ \\
\hline Linezolid & $9(100 \%)$ & $14(100 \%)$ \\
\hline Vancomcin & $9(100 \%)$ & $14(100 \%)$ \\
\hline
\end{tabular}

\section{Discussion}

Surgical site infection (SSI) is a major cause of morbidity and thus an important determinant for the success or failure of surgical practice. Given that the overall number of surgical procedures is increasing, the burden of surgical site infection (SSI) could be more significant and should merit further attention. The situation could be further aggravated due to the related problems of antimicrobial resistance ${ }^{[3]}$.

Inspite of careful antiseptic policies adopted in surgical practice, yet SSI occurs in patients and a number of exogenous and endogenous factors contribute in the incidence of these infections. In the current study, 63 patients with post operative wound infections who had undergone abdominal surgeries like cesarean section, hysterectomy, appendicectomy,

herniotomy, cholecystectomy and pyeloplasty in and around Puducherry who attended our hospital were studied. Majority of the post operative wound infection were cesarean section $25(40 \%)$. This increased prevalence in caesarean section could be due to increasing number of cases as compared to others.

Determining the microbial prevalence is of utmost important to appropriately guide antimicrobial therapy ${ }^{[11]}$. In the current study among the 63 post operative wound infection only $50(79.4 \%)$ were culture positive.

It has been regularly noted that $\mathrm{S}$. aureus continues to be the single most important bacterial species in the primary aetiology of surgical site infections since the past thirty years or so ${ }^{[12]}$. In our current study also the most frequently isolated organism was Staphylococcus aureus 26 (36.5\%). This was in accordance with other study by Krishnan et al, Ballari, Karnataka ${ }^{[8]}$, where they have also isolated $\mathrm{S}$. aureus in $31.3 \%$. In addition, prior studies in India by Surange et al ${ }^{[13]}$, Subramanian et al ${ }^{[14]}$ and recent study by Kownhar et al ${ }^{[15]}$ have stated the occurrence of S. aureus to be $34.2 \%, 30.9 \%$ and $37 \%$ respectively. Past studies revealed that $80 \%$ of healthy individuals harbor S.aureus in 
their skin or anterior nares and hence causes post operative wound infection when integrity of the skin is breached during surgery ${ }^{[16]}$.

The management of SSI caused by S.aureus is further complicated due to the emerging multi drug resistant strains, particularly the methicillin resistant S.aureus (MRSA), which also represents the leading cause of S.aureus associated nosocomial infections ${ }^{[2]}$. MRSA has become a hospital superbug throughout the world ${ }^{[16]}$. In the current study $39.1 \%$ of S.aureus isolates were methicillin resistant. Their prevalence varied from $15.7 \%$ to $63.5 \%$ in studies done in India and other countries. This marked variation could be due to preoperative and postoperative antibiotic policies and surveillance programmes adopted among different regions ${ }^{[16]}$.

The treatment of MRSA infections are further complicated as they are also usually resistant to other anti-staphylococcal agents (clindamycin, erythromycin, tetracycline, sometimes gentamicin and trimethoprim/ Sulphomethoxazole), with the exception of vancomycin [12]. In our study MRSA isolates showed decreased sensitivity to ciprofloxacin $(22.2 \%)$, erythromycin $(33.3 \%)$ and cotrimoxazole (44.4\%). Multidrug resistance seen in MRSA is multifactorial, 1. mec A gene that encodes for protein PBP-2a that binds to available lactam, 2. production of Staphylococcal pencillinase and other enzymatic deactivators, 3.presence of other resistance factors e.g. could be plasmids involvement ${ }^{[17]}$. However $88.9 \%$ sensitivity was there for amikacin and $77.8 \%$ for clndamycin. Also on the other hand all isolates both MRSA and MSSA were sensitive to linezolid and vancomycin $100 \%$.

We also compared MRSA isolates with other MSSA isolates with regard to the sensitivity pattern among the antibiotics and the resistance rates for MRSA isolates were higher than MSSA for all the antibiotics. Ranjan KP et al ${ }^{[18]}$ also observed in their study that multidrug resistance was more common in MRSA than MSSA.

\section{Conclusion}

Our current study concludes surgical site infection is a major cause of increased morbidity and a huge burden for both the patients and the surgeon. $S$. aureus continues to be the notorious organism causing SSI and also highlights clindamicin and amikacin should be used for empirical therapy preferably over cefriaxone and ciprofloxacin.

Declaration of Interest: The authors report no conflicts of interest. The authors alone are responsible for the content and writing of the paper.
Submission Declaration: This submission has not been published anywhere previously and that it is not simultaneously being considered for any other publication.

Ethical Approval: Approved by Ethical Research Committees (ERC)

\section{Reference}

1. Azoury SC, Farrow NE, Hu QL, Soares KC, Hicks CW, et al. Postoperative Wound Infections - Epidemiology, Risk Factors, Identification And Management. Chronic Wound Care Management and Research. 2015; 2:137-148.

2. Saadatian-Elahi M, Teyssou R, Vanhems P. Staphylococcus aureus, the major pathogen in orthopaedic and cardiac surgical site infections: a literature review. Int J Surg. 2008; 6:238-245.

3. Kontra JM. Reduction of surgical site infections by preoperative eradication of the staphylococcus aureus carrier state: a paradigm whose time has come. J Lancaster General Health. Spring 2012; 7(1):4-7.

4. Howard RJ,Ravitch MM, Steichen FM. Host against infections.Current problems in surgery. New English Journal of Medicine. 1980; 12:1823-1830.

5. B.B Sarkar. Post-operative infections: Physician's' perspectives.`Medicine|'Update. 2012; 22:67-71.

6. Mangram AJ, Horan TC, Pearson ML, Silver LC, Jarvis WR. Guideline for Prevention of Surgical Site Infection, 1999. Centers for Disease Control and Prevention (CDC) Hospital Infection Control Practices Advisory Committee. Am J Infect Control. 1999; 27:97-132.

7. Razavi M, Shepard DS, Suaya JA, Stason WB. Postoperative Staphylococcus aureus Infections in Medicare Beneficiaries. PLoS ONE. 2014; 9(11): 1-10.

8. Krishna S, Divya P, Shafiyabi S. Postoperative surgical wound infections with special reference to methicillin resistant Staphylococcus aureus: an experience from VIMS hospital, Ballari. J Biosci Tech. 2015; 6(3):697-702

9. Winn W, Allen S, Janda W, Koneman E, Procop G, Schreckenberger P, et al. Eds. In: Koneman's Colour Atlas and TextBook of Diagnostic Microbiology. 6th ed. Lippincott, Williams and Wilkins. 2006:643-648.

10. Performance standards for antimicrobial susceptibility testing; 17th informational supplement. CLSI M100-S17. CLSI 2007, Wayne, PA.27 (1).

11. Chahoud J, Kanafani Z, Kanj SS. Surgical site infections following spine surgery: eliminating the controversies in the diagnosis. Front Med. 2014; 1:1-10.

12. Gayathree Naik, Shrinivas Deshpande. A Study on Surgical Site Infections Caused by Staphylococcus Aureus with a Special Search for Methicillin-Resistant Isolates. Journal of Clinical and Diagnostic Research. 2011; 5:502-508.

13. Surange BN, Rai UK: Bacteriological Pattern and their sensitivity to antibiotics in Wound Infections. Ind. J. Path. Micro. 1979; 22; 331-36. 
14. Subramanian K.A, Prakash A, Shrinvas, Bhujwal RA. Postoperative wound infection. Ind J Surg. 1973; 57-64

15. Kownhar H, Shanker EM, Vignesh R, Sekar R, Velu V, Rao VA. High rate of isolation of S.aureus from Surgical Site Infections in an Indian Hospital. J Antimicrobial Chemotherapy. 2008; 3: 758-760.

16. Bhattacharya S, Pal K, Jain S, Chatterjee SS, Konar J. Surgical site infection by methicillin resistant Staphylococcus aureus - on decline? Journal of Clinical and Diagnostic Research. 2016; 10(9):DC32-DC36.
17. Batabyal B, Biswas S, Chakraborty S, Desai P and Sarkar ND, Prevalence and drug sensitivity pattern of Staphylococcus aureus in post-operative Surgical oral \& maxillofacial infections. Int. j. life sci. pharma res. 2012; 2(4): 154-158.

18. Ranjan KP, Ranjan N, Gandhi S. Surgical site infections with special reference to methicillin resistant Staphylococcus aureus: experience from a tertiary care referral hospital in North India. Int J Res Med Sci. 2013; 1(2):108-11.

\section{*Corresponding author:}

Dr. Latha Ragunathan, Professor, Department of Microbiology, Aarupadai Veedu Medical College \& Hospital, Puducherry.

Phone: +91 9894155330

Email: latha.ragunathan@avmc.edu.in

Financial or other Competing Interests: None. 